\title{
Design of a Magneto- Caloric Refrigeration System using Gadolinium Metal Powder
}

\author{
Nixson $\mathrm{M}^{\mathrm{a}}$, Paul Stephen $\mathrm{M}^{\mathrm{a}}$, \\ Sam Clitus $\mathrm{S}^{\mathrm{a}}$, Sharoon $\mathrm{J}^{\mathrm{a}}$ \\ a Department of Mechanical Engineering, \\ Loyola-ICAM College of Engineering and Technology \\ (LICET), Chennai, India
}

\author{
James Deepak ${ }^{b}$ \\ b Assistant Professor, \\ Department of Mechanical Engineering, \\ Loyola-ICAM College of Engineering and Technology \\ (LICET), Chennai, India
}

\begin{abstract}
With increase in average temperature of earth's surface temperature, air conditioners and refrigerators are indispensable. Our main and motive of this paper is to try and reduce the contribution of $R \& A C$ system to global warming by designing an eco- friendly refrigeration system. We are using Magneto- caloric effect, a phenomenon exhibited by certain rare earth metals, to bring about the cooling effect. This paper also evaluates the outcome of prototype, designed to make use of this phenomenon.
\end{abstract}

Keywords-Refrigeration; magneto caloric effect; Ecofriendly refrigeration; Gadolinium metal; Magnetic refrigeration.

\section{INTRODUCTION}

The average temperature of earth is rising at an alarming rate of $0.2^{\circ} \mathrm{C}$ per decade. Twentieth century has seen a significant achievement by the invention of airconditioners. It made our lives easier and comfortable. As every invention, it also has its dark side. Ironically, the cold air is heating the world. Air- conditioners and refrigerators contribute to global warming and climate change by emitting greenhouse gases. They also contribute to $15 \%$ of the total power consumption of the world. Air- conditioners are indispensable in our age. HVAC systems has seen many advancements till date. Their emission is much smaller now when compared to earlier days. Unfortunately, this is not enough. The global warming and climate are so impactful these days and we can and should use every possible way to reduce the emission. In order to mitigate these effects, concept called magneto-caloric refrigeration has been brought up. Basically, this refrigeration works with the aid of certain materials called magneto-caloric materials which can heat or cool with the presence and absence of magnets respectively. This article is going to talk about the working of magneto- caloric refrigeration and design process of a prototype that works with this principle and its advantages over the conventional refrigerators.

\section{MAGNETO CALORIC EFFECT}

Magneto caloric effect is a magneto- thermodynamic aspect. It is exhibited by certain rare transition elements which has the magnetic phase transition near our temperature range of interest. These materials are capable of heating up under the presence of magnetic field. This heating is attributed to the decrease of magnetic entropy and increase of orderliness within the structure i.e., the magnetic dipoles arrange themselves along the field. That is, on application of magnetic field, the dipoles arrange themselves in the direction of the field, losing their magnetic entropy. This loss of magnetic entropy causes the material to heat up as magnetic entropy is converted into thermal entropy.

Subsequently on removal of magnetic field, the magnetic dipoles ought to lose energy and randomize, which is the case in most metals. But it is not so in the case of magneto caloric materials. Their magnetic dipoles tend to remain in the same state of orientation, even after the removal of magnetic flux. This means that the material has to lose energy in some other fashion which is nothing but the loss of thermal entropy. Thus, on removal of magnetic field, the magnetic dipoles still remain in the same state of orderliness at the expense of internal heat energy of the material, and hence lowering the net temperature of the material which can reach up to $0.4 \mathrm{~K}$ at times.

\section{MAGNETO-CALORIC MATERIALS}

Many researches have been going on this field, as this Magneto caloric effect forms the basis for Magnetic refrigeration. Many magnetic solids exhibit magnetocaloric effect. But only those metals that have magnetic phase transition temperature near room temperature are quite usable for our application. One of the most notable examples of metal such magneto caloric effect near temperature is the chemical element Gadolinium and some of its alloys. Gadolinium alloys exhibit giant magneto caloric effect at near room temperature making it the best candidate for the system. Gadolinium's temperature increases when it enters certain magnetic fields. When it leaves the magnetic field, the temperature drops. The effect is considerably stronger for the gadolinium alloy $\left(\mathrm{Gd}_{5} \mathrm{Si}_{2} \mathrm{Ge}_{2}\right)$ at room temperature range. The effect of various alloy combination and as native element is studied by many scholars these days as magnetic refrigeration depends on this element mainly.

\section{REFRIGERATION CYCLE AND WORKING}

Most of the magnetic refrigeration system that works with magnetocaloric effect is based on this cycle as this is the most efficient and simple cycle to model

\section{A. Adiabatic magnetization:}

A magneto caloric substance is placed in an insulated environment. The increasing external magnetic field causes the magnetic dipoles of the atoms to align, 
thereby decreasing the material's magnetic entropy and heat capacity. The net result is that the substance is heated $(T+$ $\left.\Delta T_{\mathrm{ad}}\right)$.

\section{B. Isomagnetic enthalpy transfer:}

The added heat can then be removed by means of refrigerant, in our case, Water. Once sufficiently cooled, the magneto caloric substance and the coolant are separated.

\section{Adiabatic demagnetization:}

The substance is returned to another adiabatic condition so the total entropy remains constant. However, this time the magnetic field is decreased, the thermal energy causes the magnetic moments to overcome the field, and thus the sample cools, i.e., an adiabatic temperature change. This is based on the fact that magnetic dipoles remain to be in their oriented state. But since the magnetic field is decreased, the material has to lose energy which is none other than the thermal energy i.e., the magnetic dipoles remains oriented at the expense of the thermal energy and hence the net temperature of the specimen decreases.

\section{Isomagnetic entropic transfer:}

The magnetic field is held constant to prevent the material from reheating. The material is placed in thermal contact with the environment to be refrigerated. Because the working material is cooler than the refrigerated environment (Cold tank), heat energy migrates into the working material. The comparison of a vapour compression cycle and the magnetocaloric refrigeration cycle is shown in Fig. 1
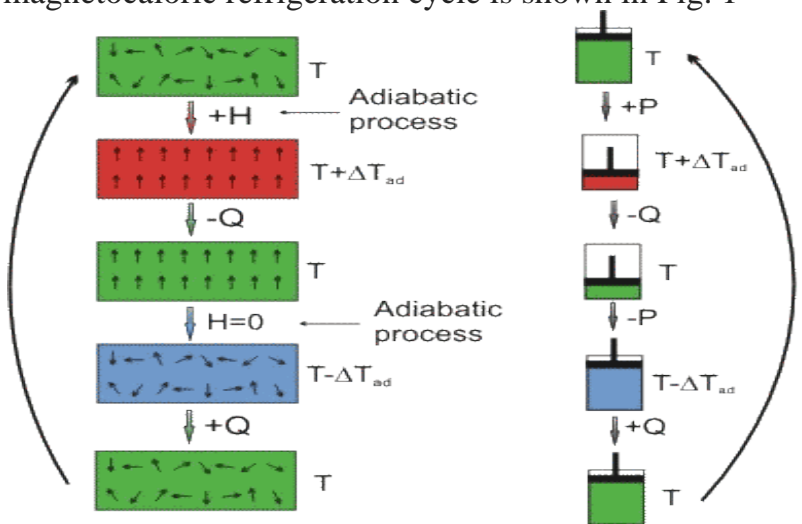

Magnetic refrigeration

Fig. 1: Refrigeration Cycle

\section{PROPOSED DESIGN}

We have tried our level best to minimize the number of moving parts in our model to reduce power consumption. All conventional systems use compressors and motors of some sort that uses power. We have designed and tested the prototype using water as the working medium and any other thermally effective liquid can also be used as long as they are eco-friendly. The only major component that uses power in our model is the electromagnets. Our primary system without the auxiliary mechanism is shown in Fig. 2.

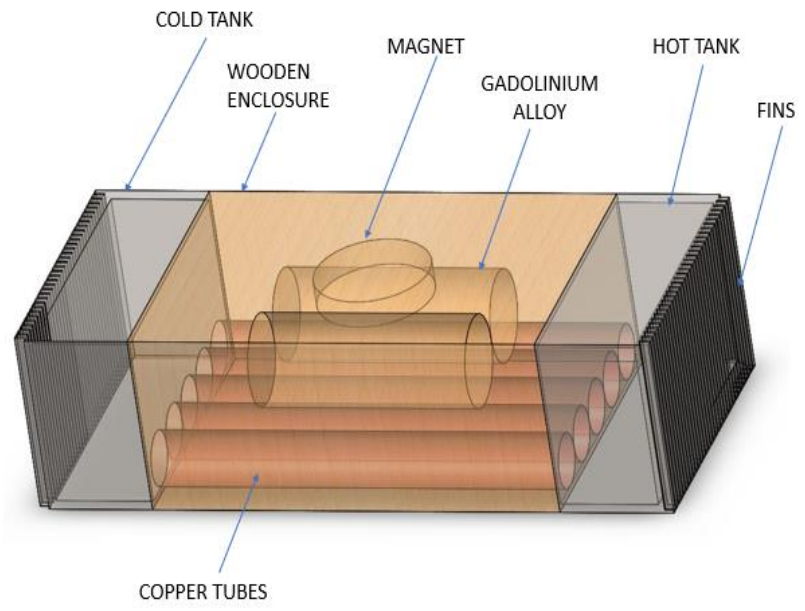

Fig. 2: Prototype Design

The main components of the system are listed below

1. Two tanks on the two ends

2. Copper tubes connecting the two tanks.

3. Aperture for placing the magnet and the gadolinium metal powder

\section{HOT AND COLD TANK:}

There are two tanks involved in the system namely hot tank and cold tank. The transfer of refrigerant from hot tank to cold tank and cold tank to hot tank will always happen and it is often referred to as cycles. The material to be chosen should have high thermal conductivity such as copper, aluminium etc. Copper is the ideal one but its cost is high compared to aluminium. For economic reasons aluminium is considered to be best even though it has thermal conductivity less than copper. These tanks are connected together by means of tubes which should also have high thermal conductivity. Fins are attached to the hot tank for carrying heat to the atmosphere. Our main area of focus is the cold tank where our inference and testing will take place

\section{MAGNETOCALORIC MATERIAL}

As we have discussed earlier, Gadolinium is the dominant among the magneto caloric materials. This material is placed between the two tanks on the tubes connecting them. and it surrounds the tube which is connecting the two tanks. The gadolinium metal should be in direct contact with the tubes. The tubes should have high thermal conductivity and thus copper tubes are chosen. Due to design constraints, gadolinium is powdered and place on the tube. In fact, MCE is more pronounced in powdered form. The gadolinium powder is not spread throughput the tube but concentrated near the magnets so that the magnetic flux passes through them without fail.

\section{MAGNET:}

There are two types of magnets which can be used for the system such as permanent magnet and electromagnet. In case of permanent magnet, the magnetic field will always be present and it has to be moved away periodically in order 
to make the change in field whereas in case of electromagnet a simple cutting of electricity periodically can change the magnetic field. But electromagnets have the disadvantage of heating up on magnetisation and their size to produce enough magnetic flux is quite huge. Considering these we went on with the choice of using a neodymium permanent magnet.

\section{Auxiliary Tilting Mechanism:}

In order to facilitate the movement of water from one tank to other. The whole setup is tilted. This tilting is brought about by an auxiliary tilting mechanism or table. The table is motor operated and the stepper motor used is calibrated so as to remain in a certain position for a particular calculated period of time.

The design specifications of the prototype are given below.

1. TANK:

- Material: Aluminium; Thermal conductivity $\mathrm{K}=205 \mathrm{~W} / \mathrm{mK}$

- Length: 40mm;

- Width: $100 \mathrm{~mm}$;

- Height: $60 \mathrm{~mm}$; Thickness of wall: $2 \mathrm{~mm}$; Volume: 0.1901

2. COPPER TUBE:

- $\mathrm{K}=385 \mathrm{~W} / \mathrm{mK}$

- Diameter:5/8 inch

- Length: 150mm (Between two tanks)

VI. WORKING OF THE SYSTEM

As said earlier, the working principle is based on Magnetocaloric effect. It was earlier studied by Mouaaz et al., that a sample of gadolinium can produce a temperature difference of about $10 \mathrm{~K}$ under a magnetic field of $5 \mathrm{~T}$.

Based on that study, a permanent magnet that is capable of producing $1 \mathrm{~T}$ is selected for the system. The disadvantage in using electromagnet is that the magnet itself heats up during magnetization and due to design constraints magnetization would not be even. In order to achieve those qualities, the electromagnet has to be as quite large. Hence, Neodymium Magnets are considered after it's capability.

The refrigeration cycle starts with the magnetization of the Gadolinium powder by the magnet. The magnet is brought in the enclosure by a pulley and motor arrangement. When the magnet is inside the enclosure, it magnetises the gadolinium specimen. This is the adiabatic magnetisation. Due to its magnetocaloric property, the magnetisation causes the material to heat up. By theory, the copper tubes would also heat up.

This heat is removed by the flow of refrigerant. Water is chosen as the refrigerant as it is the most ecofriendly liquid one can ever get. This is an experimental liquid and it can always change with further development. The liquid is passed from cold tank (on the left) to the hot tank through copper tubes by tilting the table towards the hot tank. Copper has the highest thermal conductivity among commercial metals available. On passing through the tube, water gets heated up. This is the Isomagnetic Enthalpy Transfer of our system. The tilting stepper motor is calibrated to produce a slow and steady tilting in order to facilitate stream line, laminar flow so that maximum heat is transferred to water. Ans so, the copper tubes and the gadolinium metal cool down.

On reaching the hot tank, the hot water starts losing its heat through the aluminium fins. The aluminium fins are also attached with a fan to aid in the cooling. Throughout these stages, magnetic field is applied.

Once the water reaches the hot tank, magnet is retracted out of the enclosure, making our gadolinium metal demagnetised. By principle, MCE material would cool down. This is the Adiabatic Demagnetisation phase.

Now water is passed from hot tank to cold tank and water is cooled to a significant level, which is nothing but the Isomagnetic Heat Transfer.

By applying $2 \mathrm{~T}$ magnetic field, a temperature change of about $4^{\circ} \mathrm{C}$ can be obtained. This change cannot be expected in a single cycle. Existing systems take minimum of 30 cycles to reach a refrigeration level cooling.

The periodic movement of liquid from cold tank to hot tank and vice versa is done by a special mechanism. The primary system is placed over this mechanism.

The feedback of the cooling effect can be identified by measuring the temperature of water in the cold tank. The temperature is measured using a thermocouple temperature sensor module.

\section{INFERENCE}

Reading are initially taken with the gadolinium powder alone

i.e., not inside the refrigeration system. This was done in order to test the effectiveness of the material and the magnet. The readings were given in Table. 1 . Time zero corresponds to time when the magnet is brought closer to attract the $\mathrm{Gd}$ powder,

\begin{tabular}{|c|c|}
\hline Time (s) & $\begin{array}{c}\text { Temperature } \\
\left({ }^{\circ} \mathrm{C}\right)\end{array}$ \\
\hline 0 & 32 \\
\hline 30 & 32.3 \\
\hline 60 & 32.8 \\
\hline 90 & 33.2 \\
\hline 120 & 33.5 \\
\hline 150 & 33.8 \\
\hline 180 & 34 \\
\hline 210 & 34.2 \\
\hline 240 & 34.3 \\
\hline 270 & 34.3 \\
\hline 300 & 34.4 \\
\hline 330 & 34.5 \\
\hline
\end{tabular}

Table. 1

The graph corresponding to this table is given in fig. 3 


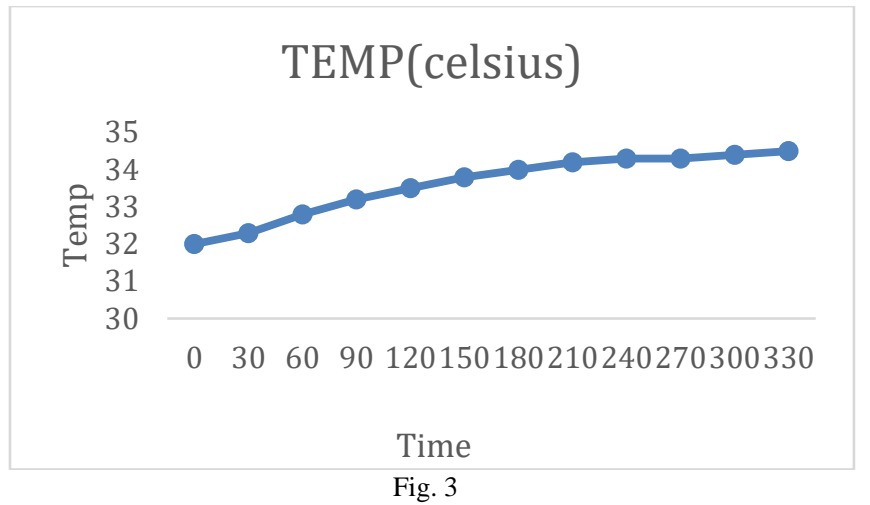

From the chart, it is clear the with the application of magnetic field, the temperature clearly rises.

The inference and readings noted with the actual system in action is given below. The cycle starts with the application of magnetic field on the gadolinium metal powder.

The magnetic field is initially applied for 60 seconds, and then the tilting table starts. The reading at Zero second corresponds to this position i.e., the start of tilting. The water now moves from cold tank to hot tank. The motor is calibrated in such a way that it takes 30 seconds for the water to move one from one tank to another and the magnet is pulled out after this takes place. The hot water stays in the hot tank for 60 seconds to facilitate cooling. And then, water starts moving to cold tank by tilting. The reaching of water in cold tank marks the completion of the cycle. The readings for five such cycles are given below in table. 2 and the corresponding graph in Fig. 4

\begin{tabular}{|c|c|}
\hline Time $(\mathrm{s})$ & Temp $\left({ }^{\circ} \mathrm{C}\right)$ \\
\hline 0 & 29.5 \\
\hline 120 & 29.4 \\
\hline 300 & 29.3 \\
\hline 480 & 29.1 \\
\hline 660 & 28.8 \\
\hline 840 & 28.6 \\
\hline
\end{tabular}

Table. 2: Time vs Temperature

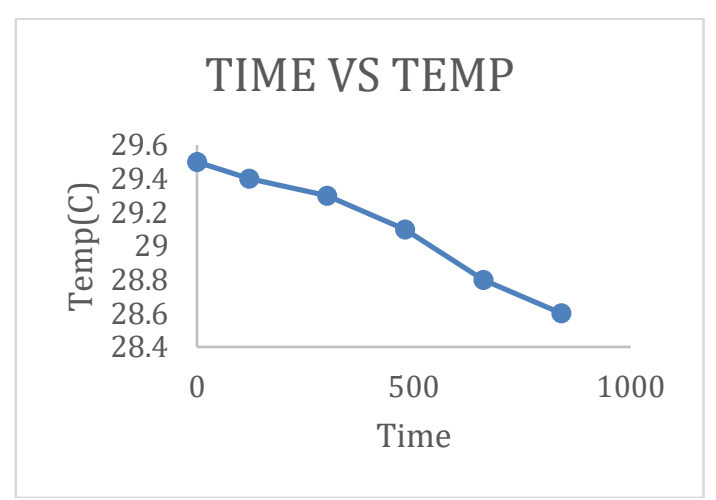

Fig. 4

All temperatures were measured using a thermocouple, temperature sensor module W1209.

From the graphs, we can infer effectiveness of gadolinium and the impact it would have on achieving less emission. The power required for this system is also very low as there are very fewer moving parts and all the parts can be coupled to a single motor. The green house emission is also reduced an we ae using only water as the working medium.

The only drawback noted in implementing this technology is the availability of Gadolinium metal. As it is a rare earth metal, the availability of the metal is very limit.

\section{CONCLUSIONS}

Magnetic refrigeration is a technology that has proven to be environmentally safe. Results have shown 30\% efficiency improvement over vapour compression systems. In order to make the Magnetic Refrigerator commercially available scientists need to know how to achieve larger temperature variations. Two advantages of using Magnetic Refrigeration system instead of vapour compression systems are no harmful refrigerants used and they can be up to $50 \%$ efficient.

With our minimal setup we were able to achieve a temperature difference of almost $1^{\circ} \mathrm{c}$, which can well be increased with a more sophisticated setup.

There are still some thermal and magnetic hysteresis problems to be solved for these first-order phase transition materials that exhibit the Magneto Caloric Effect to become really useful.

\section{REFERENCES}

[1] H. Auracher, P.w. Egolf, ' Magnetic Refrigeration at Room Temperature'; International Journal of Refrigeration vol 29, issue 8, pages 1235-1394. (Dec 2006).

[2] K.A. Gschneidner, Jr. V.K. Pecharsky,' Thirty years of near room temperature magnetic cooling: Where we are today and future prospects'; International journal of refrigeration 31(2008) $945-$ 961.

[3] Y Mozharivskyj, ' Magnetocaloric Effect and Magnetocaloric Materials'; Reference Module in Chemistry, Molecular Sciences and Chemical Engineering.

[4] Houssem Rafik El-Hana Bouchekara and Mouaaz Nahas, 'Magnetic Refrigeration Technology at Room Temperature'; Trends in Electromagnetism - From Fundamentals to Applications.

[5] Behzad Monfared, Bjorn Palm, 'Material requirement for magnetic refrigeration applications'; International journal of refrigeration vol 96, Pages 25-37. (Dec 2018).

[6] C.Aprea, A. Greco et al, 'Magnetic refrigeration: A Promising new technology for energy saving'; International Journal of Ambient energy, vol 37, issue 3, (14 Oct 2014). 